\title{
Experimental SEFDM Pipelined Iterative Detection Architecture with Improved Throughput
}

\author{
Waseem Ozan ${ }^{1}$, Paul Anthony Haigh ${ }^{1}$, Bo Tan ${ }^{2}$ and Izzat Darwazeh ${ }^{1}$ \\ ${ }^{1}$ Department of Electronic and Electrical Engineering, University College London, London, UK, WC1E 6BT \\ ${ }^{2}$ School of Computing, Electronics and Mathematics, Coventry University, Coventry, UK \\ ${ }^{1}$ \{w.ozan; p.haigh; i.darwazeh\}@ucl.ac.uk, ${ }^{2}$ bo.tan@ coventry.ac.uk
}

\begin{abstract}
In spectrally efficient frequency division multiplexing (SEFDM), the separation between subcarriers is reduced below the Nyquist criteria, enhancing bandwidth utilisation in comparison to orthogonal frequency division multiplexing (OFDM). This leads to self-induced inter-carrier interference (ICI) in the SEFDM signal, which requires more sophisticated detectors to retrieve the transmitted data. In previous work, iterative detectors (IDs) have been used to recover the SEFDM signal after processing a certain number of iterations, however, the sequential iterative process increases the processing time with the number of iterations, leading to throughput reduction. In this work, ID pipelining is designed and implemented in software defined radio (SDR) to reduce the overall system detection latency and improve the throughput. Thus, symbols are allocated into parallel IDs that have no waiting time as they are received. Our experimental findings show that throughput will improve linearly with the number of the paralleled ID elements, however, hardware complexity also increases linearly with the number of ID elements.
\end{abstract}

Index Terms-Iterative detector, non-orthogonal, pipelining design, spectrally efficient, SEFDM, OFDM.

\section{INTRODUCTION}

Spectrally efficient frequency division multiplexing (SEFDM) is a research topic that is becoming the focus of a great deal of interest in recent years [1-6], within the context of non-orthogonal modulation formats, which are gaining popularity for 5G systems [7]. Such interest in SEFDM derives from its ability to save spectrum in comparison to orthogonal frequency division multiplexing (OFDM) by breaking the orthogonality of the subcarriers, placing them at frequencies that are below the symbol rate [8]. This is timely due to the almost exponentially increasing amount of mobile data traffic [9], expected to exceed $24.3 \mathrm{~EB} /$ month by 2019 . This will only be compounded by the worldwide roll-out of 5th generation networks in 2020, where data traffic is expected to increase significantly. It is well known that radio spectrum is already heavily subscribed, leading to high-cost premiums, and as such, modulation formats such as SEFDM that save spectrum are highly sought after.

Research into non-orthogonal modulation has increased rapidly over the last decade, with several candidate technologies proposed in the literature, with alternatives to SEFDM including faster-than-Nyquist (FTN) pulse shaping [10], and truncated-OFDM (TOFDM) [11], amongst others [12,13]. FTN is a time-domain technique that reduces the transmission period for each symbol, thus improving spectral efficiency. On the other hand, TOFDM increases the transmission speed by partial transmission of OFDM symbols in the time-domain.

SEFDM operates slightly differently from FTN and TOFDM, however, and is a frequency-domain system that saves bandwidth by compressing its symbols in frequency, and this bandwidth gain translates directly into a capacity gain. The bandwidth compression factor is usually denoted $\alpha$, where $(1-\alpha) \times 100 \%$ is the amount of bandwidth saved, in comparison to traditional OFDM for an equivalent number of bits. Incidentally, when $\alpha=1$, there is no compression and OFDM is transmitted [8].

SEFDM is not without disadvantages though, and one of the most significant is the computational complexity requirements of the receiver [14]. Normally, sphere decoders (SDs) are utilised to undo the self-induced inter-carrier interference (ICI) experienced by exceeding the orthogonality limits of subcarrier spacing [15]. Alternatively, iterative detectors (IDs) have been demonstrated in the literature which are relatively low complexity in comparison [16] and [2], but introduce significant latency due to their iterative nature. In this paper, for the first time, we propose a pipelined ID structure to increase throughput at the cost of additional computational complexity. We demonstrate that with no loss in performance in comparison to traditional implementations of SEFDM with an ID, throughput can be increased linearly with the number of pipelined stages.

\section{SEFDM Signal MODEL}

The SEFDM signal consists of $N$ non-orthogonal subcarriers, and each one carries a complex signal, denoted by $s$. The SEFDM signal, $x(t)$, consisting of $m$ SEFDM data symbols, is represented in the continuous time-domain as:

$$
x(t)=\frac{1}{\sqrt{T}} \sum_{m=-\infty}^{\infty} \sum_{n=0}^{N-1} s_{m, n} \exp \left[\frac{j 2 \pi n \alpha(t-m T)}{T}\right]
$$

where $T$ is the period of an SEFDM symbol, $\alpha<1$ is the bandwidth compression factor, $N$ is the number of subcarriers in every symbol, and $s_{m, n}$ is the complex symbol modulated on the $n^{t h}$ subcarrier belonging to the $m^{t h}$ SEFDM symbol.

In the discrete time-domain, the same SEFDM symbol can be represented in matrix form as follows [5]:

$$
X=\mathbf{\Phi} S
$$

where $X$ represents a $Q$-dimensional vector of a sampled SEFDM symbol in the time-domain, $S$ is an $N$-dimensional 
vector of a sampled input signal in the frequency-domain and $\boldsymbol{\Phi}$ is a $Q \times N$ two-dimensional matrix that signifies the sampled carrier matrix [5].

Consider that the transmitted SEFDM symbols pass through a wireless fading channel $\boldsymbol{H}$, which leads to a channeldistorted signal contaminated by noise $Z$, resulting in the received signal to be demodulated. The reception process is expressed as follows:

$$
R=\boldsymbol{\Phi}^{*} \boldsymbol{H} X+\boldsymbol{\Phi}^{*} Z=\boldsymbol{\Phi}^{*} \boldsymbol{H} \boldsymbol{\Phi} S+\boldsymbol{\Phi}^{*} Z
$$

where $R$ is the demodulated signal consisting of a vector of symbols of length $N$ and $(.)^{*}$ is the transpose conjugate operation.

\section{TESTBED Descriptions}

The software and hardware designs of the real-time experiment are presented in this section to evaluate SEFDM systems in Long Term Evolution (LTE) Extended Pedestrian A (EPA) channel model. A photograph of the experimental testbed is shown in Fig. 1. The experimental testbed contains several universal software radio peripheral (USRP) transceivers (NI USRP RIO N2395R) programmed using LabVIEW and a Spirent VR5 channel emulator to generate realistic LTE channels. The software design of signal generation and transmission, signal synchronisation, channel estimation and equalisation, iterative signal detection and the new pipeline processing method, all developed in real time on the USRPs are detailed below.

\section{A. Transmission}

At the transmitter, a pseudorandom binary sequence is generated, which is then encoded by a recursive convolutional coder with code rate $R_{c}=1 / 2$, forward polynomial $G 1=$ $1+D+D^{2}$ and feedback polynomial $G 2=1+D^{2}[2,16]$. The coded bits are then interleaved by a block interleaver before being mapped onto the appropriate constellation. In this work, we test binary phase shift keying (BPSK), quadrature phase shift keying (QPSK) and 8-phase shift keying (8-PSK). Next, the symbols are converted into a parallel stream which feeds an inverse fast Fourier transform (IFFT), resulting in the generation of SEFDM symbols. The distance between subcarriers is compressed by a factor $\alpha \leq 1$, where $\alpha=1$ for OFDM. The SEFDM symbols are then converted back into serial streams by a parallel-to-serial $(P / S)$ converter. In order to decrease the effect of inter-symbol interference

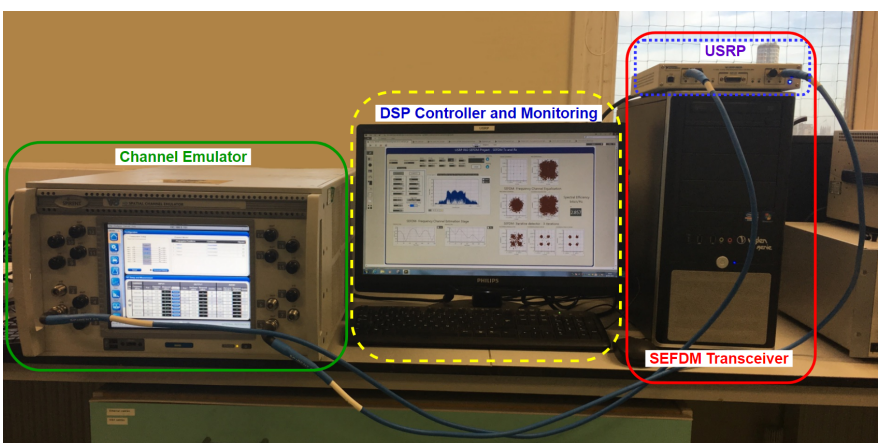

Fig. 1. SEFDM transceiver test-bed setup
(ISI) between adjacent symbols in a realistic wireless channel, a cyclic prefix (CP) is added at the beginning of every transmitted symbol. In the final stage of the transmitter, the complex SEFDM signal is fed to the FPGA that drives the USRP RIO, before digital-to-analogue conversion (DAC) and up-conversion by a local oscillator running at $2 \mathrm{GHz}$. Table I depicts the system parameters used in this experiment.

TABLE I

EXPERIMENTAL SYSTEM SPECIFICATIONS

\begin{tabular}{ll}
\hline Parameters & Values \\
\hline \hline Central carrier frequency & $2 \mathrm{GHz}$ \\
\hline Sampling frequency & $30.72 \mathrm{MHz}$ \\
\hline Signal bandwidth & $18 \mathrm{MHz}$ \\
\hline Values of $\alpha$ & $1(\mathrm{OFDM}) ; 0.9 ; \ldots ; 0.4$ \\
\hline Subcarrier baseband bandwidth & $60 \mathrm{KHz}$ \\
\hline Subcarrier spacing & $\alpha \times 60 \mathrm{KHz}$ \\
\hline IFFT/FFT size & 512 \\
\hline Cyclic prefix & 128 time samples \\
\hline Modulation scheme & BPSK; QPSK; 8-PSK \\
\hline
\end{tabular}

\section{B. LTE Fading Channel Model and Signal Synchronisation}

The radio frequency (RF) signal is transmitted through the VR5 channel emulator that has LTE EPA5 wireless channel model [17], and is set using the parameters shown in Table II. The output of the VR5 channel emulator is fed back to the receiver of the USRP device, which down-converts the RF signal to the baseband, before analogue-to-digital conversion. A Schmidl and Cox [18] synchronisation is applied in this experiment, where two identical timing sequences are added at the start of each frame to estimate the first sample of the data symbols.

TABLE II

LTE EPA FADING CHANNEL MODEL

\begin{tabular}{|c|c|c|c|}
\hline Path & Doppler frequency & Relative power & Delay values \\
\hline 1 & $5 \mathrm{~Hz}$ & $0.0 \mathrm{~dB}$ & $0 \mathrm{~ns}$ \\
\hline 2 & $5 \mathrm{~Hz}$ & $-1.0 \mathrm{~dB}$ & $30 \mathrm{~ns}$ \\
\hline 3 & $5 \mathrm{~Hz}$ & $-2.0 \mathrm{~dB}$ & $70 \mathrm{~ns}$ \\
\hline 4 & $5 \mathrm{~Hz}$ & $-3.0 \mathrm{~dB}$ & $90 \mathrm{~ns}$ \\
\hline 5 & $5 \mathrm{~Hz}$ & $-8.0 \mathrm{~dB}$ & $110 \mathrm{~ns}$ \\
\hline 6 & $5 \mathrm{~Hz}$ & $-17.2 \mathrm{~dB}$ & $190 \mathrm{~ns}$ \\
\hline 7 & $5 \mathrm{~Hz}$ & $-20.8 \mathrm{~dB}$ & $410 \mathrm{~ns}$ \\
\hline
\end{tabular}

\section{Channel Estimation/Equalisation}

In this work, the pilot is sent as an OFDM symbol, but at a lower rate in comparison to SEFDM symbols [19]. Hence, we design our OFDM pilot such that the subcarrier frequencies are equivalent to those of the SEFDM subcarriers, but without the inter-carrier interference, since these pilots are orthogonal. This allows the use of a simple one-tap equaliser in the frequency domain to mitigate the effect of the channel. The $\mathrm{CP}$ is then removed from the received symbols, and the first symbol of $r$ (i.e. the pilot symbol) is fed to the channel estimator, which is then used for channel equalisation to mitigate the phase and amplitude distortion on the signal. 


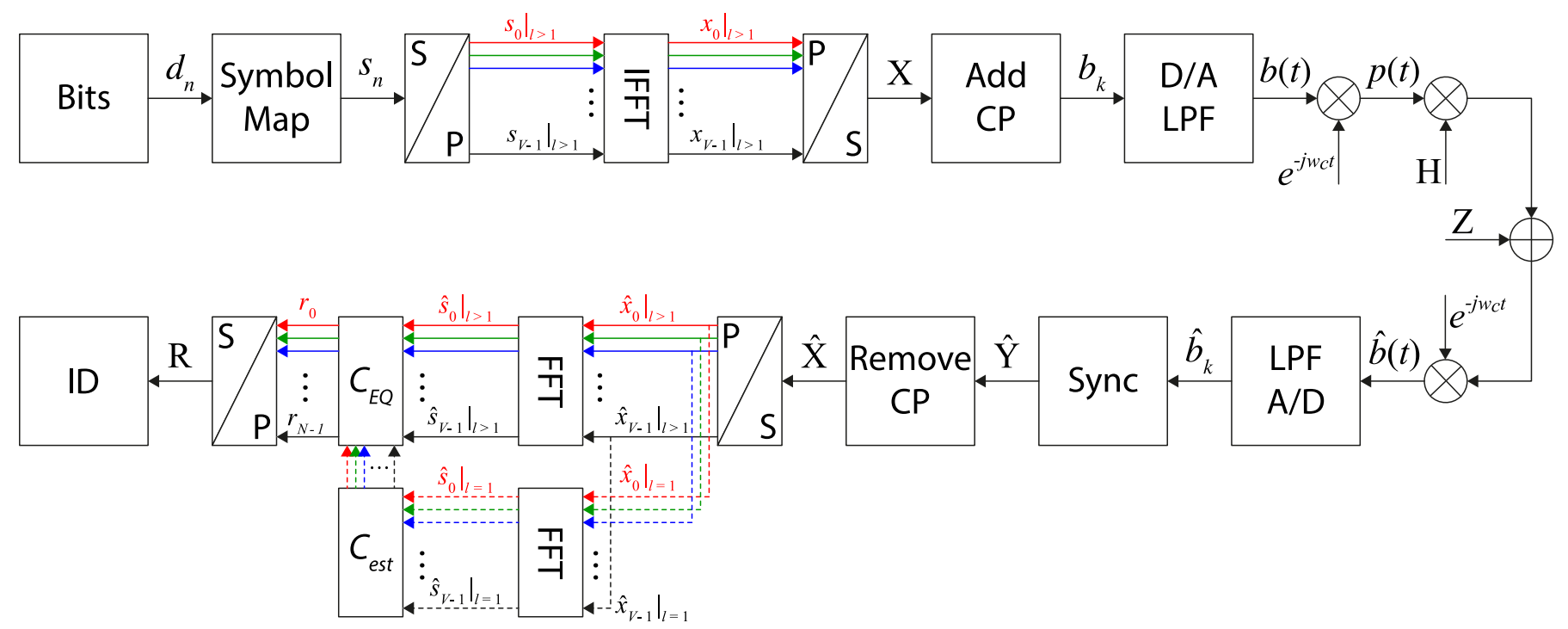

Fig. 2. Schematic block diagram of SEFDM system

\section{Signal Detection}

To recover the transmitted signal, we implement an ID based on the turbo equalisation technique with an interference canceller which is fully detailed in $[2,16]$. In every iteration, the interference between the subcarriers is estimated and subtracted from the original received signal $R$, before being passed to the next iteration.

The equalised data is subsequently de-mapped and deinterleaved at the beginning of each iteration before Viterbi decoding. Using the estimated correlation matrix and the decoded data, the interference generated between SEFDM subcarriers is estimated. After subtracting the estimated interference from the received signal, the result is passed back into the decoding process to improve the interference cancellation; repetition of this process leads to a better estimate of the transmitted data.

\section{E. Pipeline Processing in SDR}

As described previously (Section III-D), the SEFDM receiver requires the ID for eliminating the inter-subcarrier interference, and one of the negative impacts of this is the introduction of a significant processing delay that limits system throughput. Thus, we introduce a pipeline processing flow on this software defined radio (SDR) testbed to improve the overall throughput of the ID. Pipelining is a well-known concept in real-time SDR processing [20] and FPGA processing flow design [21]. In this work we adopt this signal processing technique by leveraging on the power of decentralised multicore processors. The proof of the pipeline design on the SDR platform provides a guideline for implementation on FPGAs. The principle of pipeline flow design is to decompose the long processing sequence into a group of sub-modules. By allocating each sub-module with new data, the pipeline mechanism maximises efficiency of computing resources by avoiding the idle/waiting status of sub-modules. In [22] and [23], FPGA designs for SEFDM transmitter and receivers were introduced respectively, then, a pipelined architecture has been proposed for SEFDM transmitters in [24]. For the SEFDM

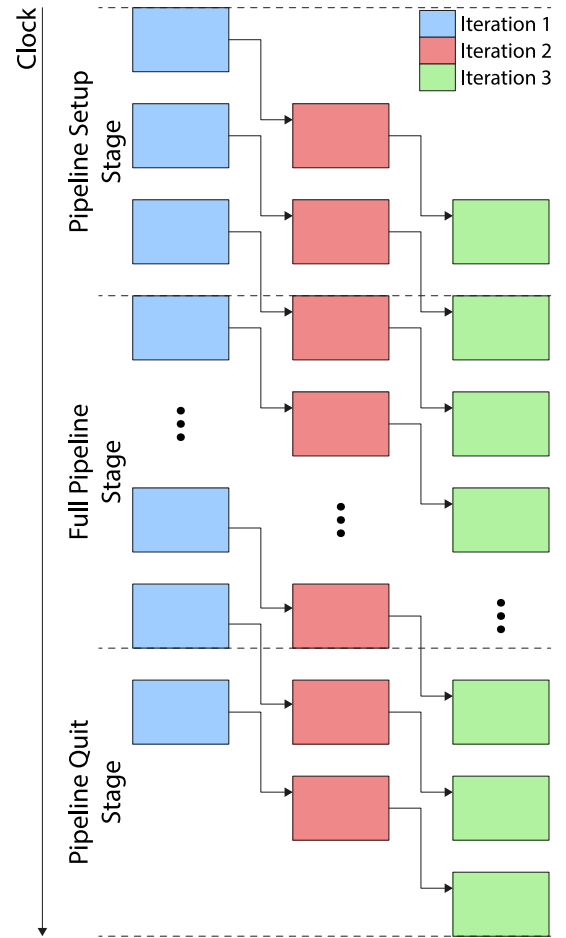

Fig. 3. Pipeline design in side of a single iteration

receiver case, this work demonstrates an example pipeline flow design ID, as illustrted in Fig. 3.

The principle for sub-module processing is to distribute evenly the processing delay to make $\max \left\{\tau_{i}\right\} \leq \tau$, where $\tau_{i}$ is the processing delay of the $i^{\text {th }}$ sub-module $\tau$ is symbol duration of SEFDM. Fig 3, shows that all the sub-modules are fully used after the pipeline setup stage. This helps to improve the system throughput significantly, up to $\eta$ times, due to the fact that the processing delays are evenly distributed in each sub-module, where $\eta$ is the number of sub-modules.

The block diagram (Fig 3) shows the pipeline flow for a single ID. Measurement in Fig. 7, a single cancellation iteration is not sufficient to mitigate residual interference from other sub-carriers. Thus, it is necessary to perform a 
certain number of iterations to suppress fully the interference, especially for low $S / N$ values.

An additional advantage of pipeline processing on the SDR is that the software environment provides sufficient flexibility and time budget for precise calculation to balance the load between the sub-modules. The tested calculation load allocation strategy can be easily transplant for FPGA pipeline design.

\section{EXPERIMENTAL RESULTS}

The measured BER of BPSK, QPSK and 8PSK-SEFDM are shown in Figs. 4, Fig. 5 and Fig. 6, respectively. Upon inspection, it is clear that each modulation format approaches the target BER after reaching the third iteration of ID for varying degrees of $\alpha$. It is also possible to infer that a higher ratio of bandwidth compression (decreasing alpha) is possible with a lower number of bits/symbol, since a value of $\alpha=0.4$ can be supported with sufficiently low BER for BPSK with a power penalty of $\sim 2 \mathrm{~dB}$. On the other hand, for QPSK, $\alpha=0.7$ can be supported with approximately equivalent performance in comparison to OFDM, as the power penalty is approximately $4 \mathrm{~dB}$. Finally, For 8-PSK, $\alpha=0.8$ is the lowest value that can be supported over the range tested, where a power penalty of $5 \mathrm{~dB}$, whereas error floors are observed for $\alpha \leq 0.7$.

In Fig. 7, constellations for $\alpha=0.7$ QPSK are shown and so is the signal spectrum. The top left constellation shows the received symbols after the FFT (referring to Fig. 2) while the top right constellation shows the same data after channel estimation and equalisation. Clearly, at this stage the data cannot be recovered successfully and hence the requirement for the ID. In Fig. 7, the progressive improvement in received signal constellation is evident as the number of iterations is increased from one (left) to three (right).

Finally, we note that the transforming of the ID into a pipelined structure will increase throughput linearly, by a factor of $\eta$, where $\eta$ is the number of stages in the structure. However, this comes at a cost of computational complexity, which also increases linearly with $\eta$. The convolutional decoder algorithm is the dominant source of computational complexity in the ID, featuring a significant number of additions, which can be calculated following [25]. In Fig. 8 we illustrate this, where it is clear that as $\eta$ increases, here we show a range of $\eta$ from 1 to 10 , the number of operations per second increases from 1,613 addition operations/second for $\eta=1$, to 16,130 for $\eta=10$. Due to the extensive capabilities of modern digital signal processing units such as field programmable gate arrays (FPGAs), we suggest that this could easily be supported without taking significant resources required for further processing.

\section{CONClusion}

In this work we have experimentally demonstrated a pipelined iterative detector structure for applications of SEFDM. We show that by processing the ID iterations in parallel, SEFDM links can be supported with $\alpha=0.4$ (BPSK), $\alpha=0.7$ (QPSK) and $\alpha=0.8$ (8-PSK), demonstrating no

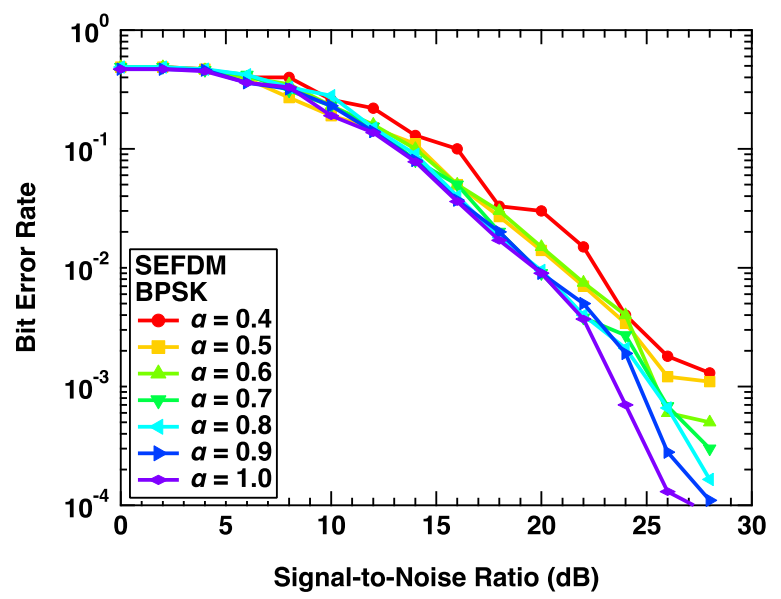

Fig. 4. BER of BPSK-SEFDM using OFDM pilots

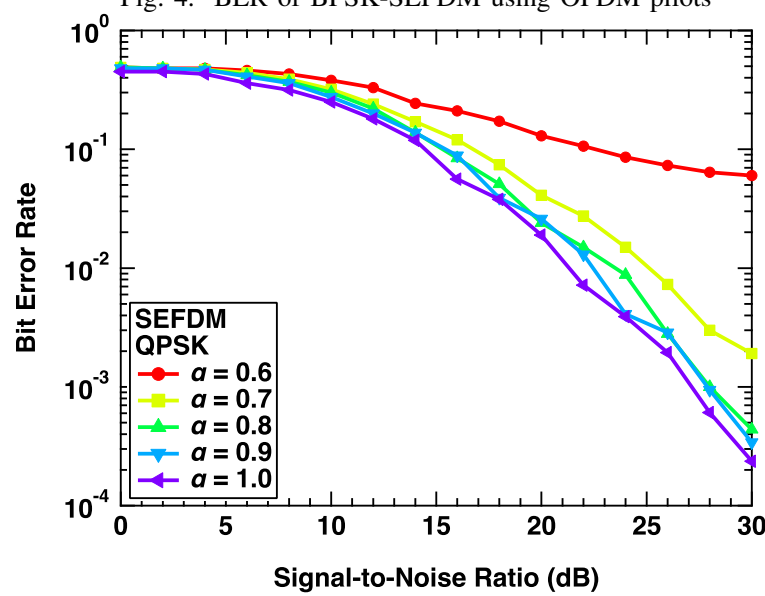

Fig. 5. BER of QPSK-SEFDM using OFDM pilots

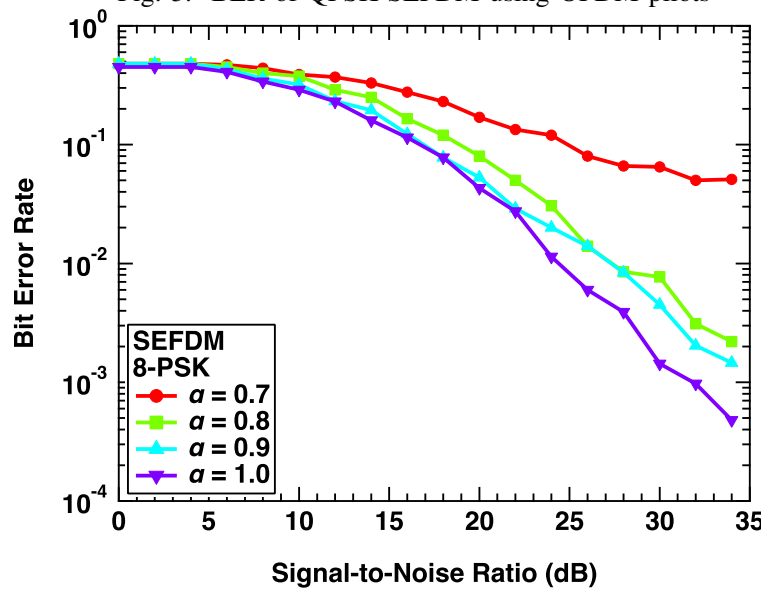

Fig. 6. BER of 8-PSK-SEFDM using OFDM pilots

loss in BER performance in comparison to traditional IDs. We further calculate the throughput improvement of the proposed, and also discuss the computational complexity. We show that computational complexity increases linearly with throughput.

\section{ACKNOWLEDGMENT}

This work was part funded by two EPSRC grants "Impact Acceleration Discovery to Use" and EP/P006280/1: MARVEL. The work was also supported by National Instruments and a donation of the LTE FPGA core through the Xilinx 

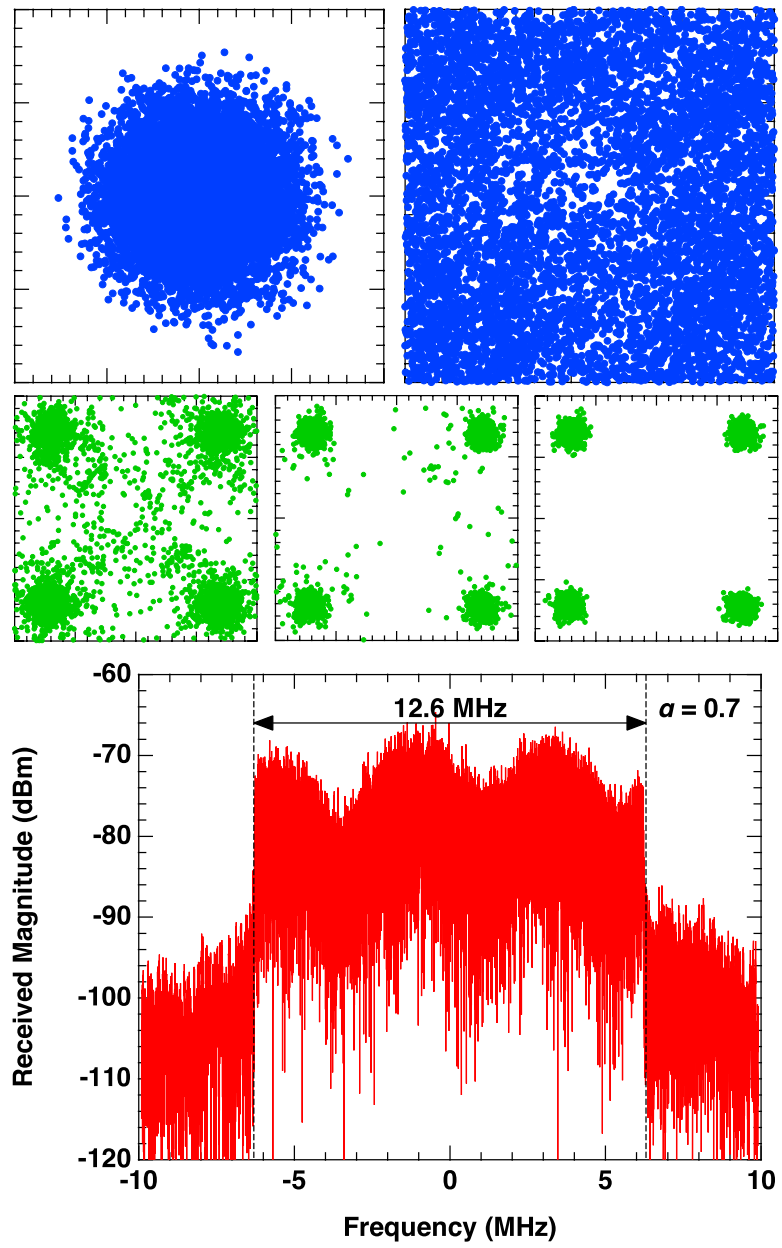

Fig. 7. Received constellations and measured spectra for $\alpha=0.7$

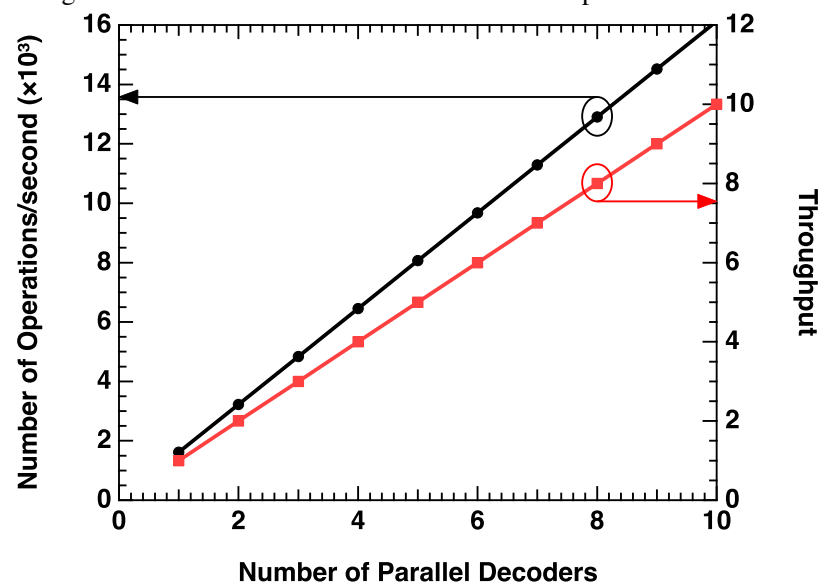

Fig. 8. Calculated computational complexity and throughput for the proposed pipelined receiver

University Donation program. We are grateful for UCL's studentship for Waseem Ozan $\mathrm{PhD}$ studies.

\section{REFERENCES}

[1] S. V. Zavjalov, S. V. Volvenko and S. B. Makarov, "A Method for Increasing the Spectral and Energy Efficiency SEFDM Signals," in IEEE Communications Letters, vol. 20, no. 12, pp. 2382-2385, Dec. 2016.

[2] K. Park, et al., "Iterative frequency-domain inter-carrier interference cancellation for coded SEFDM," in Electronics Letters, vol. 53, no. 19, pp. 1333-1335, 9142017.
[3] D. Rainnie, et al., On capacity merits of spectrally efficient FDM, in Military Communications Conference, IEEE MILCOM 2015, pp. 581586.

[4] Y. Wang, et al., "SEFDM Based Spectrum Compressed VLC System Using RLS Time-domain Channel Estimation and ID-FSD Hybrid Decoder," On capacity merits of spectrally efficient FDMECOC 2016; 42nd European Conference on Optical Communication, Dusseldorf, Germany, 2016, pp. 1-3.

[5] T. Xu and I. Darwazeh, "Transmission Experiment of Bandwidth Compressed Carrier Aggregation in a Realistic Fading Channel," in IEEE Transactions on Vehicular Technology, 2017.

[6] J. Fan, et al., "Faster-Than-Nyquist Signaling: An Overview," in IEEE Access, vol. 5, pp. 1925-1940, 2017.

[7] F.-L. Luo and C. Zhang, Signal Processing for 5G: Algorithms and Implementations. Wiley, 2016.

[8] I. Kanaras, et al., "Spectrally Efficient FDM Signals: Bandwidth Gain at the Expense of Receiver Complexity", in IEEE International Conference on Communications (ICC), Dresden, 2009, pp. 1-6.

[9] -, "Cisco Visual Network Index: Global Mobile Data Traffic Forecast (2016-2021)", Cisco Public Information White Paper, 2017.

[10] J. B. Anderson, F. Rusek and V. wall, "Faster-Than-Nyquist Signaling," in Proceedings of the IEEE, vol. 101, no. 8, pp. 1817-1830, Aug. 2013

[11] W. Ozan, K. Jamieson and I. Darwazeh, "Truncating and oversampling OFDM signals in white Gaussian noise channels," 2016 10th International Symposium on Communication Systems, Networks and Digital Signal Processing (CSNDSP), Prague, 2016, pp. 1-6.

[12] Renyuan Li and G. Stette, "Time-limited orthogonal multicarrier modulation schemes," in IEEE Transactions on Communications, vol. 43, no. 2/3/4, pp. 1269-1272, Feb./March/April 1995.

[13] M. Hamamura and S. Tachikawa, "Bandwidth efficiency improvement for multi-carrier systems," 2004 IEEE 15th International Symposium on Personal, Indoor and Mobile Radio Communications (IEEE Cat. No.04TH8754), 2004, pp. 48-52 Vol.1.

[14] I. Kanaras, et al., "Investigation of a Semidefinite Programming detection for a spectrally efficient FDM system," 2009 IEEE 20th International Symposium on Personal, Indoor and Mobile Radio Communications, Tokyo, 2009, pp. 2827-2832.

[15] S. Isam and I. Darwazeh, "Design and Performance Assessment of Fixed Complexity Spectrally Efficient FDM Receivers," 2011 IEEE Vehicular Technology Conference (VTC Spring), 2011.

[16] T. Xu and I. Darwazeh, "A Soft Detector for Spectrally Efficient Systems With Non-Orthogonal Overlapped Sub-Carriers," in IEEE Communications Letters, vol. 18, no. 10, pp. 1847-1850, Oct. 2014.

[17] Evolved Universal Terrestrial Radio Access (E-UTRA); Base station radio transmission and reception, 3GPP TS 36.104 V10.2.0, May 2011

[18] T. M. Schmidl and D. C. Cox, "Robust frequency and timing synchronization for OFDM," in IEEE Transactions on Communications, vol. 45, no. 12, pp. 1613-1621, Dec 1997.

[19] W. Ozan, H. Ghannam, P. A. Haigh and I. Darwazeh, "Experimental implementation of real-rime non-orthogonal multi-carrier systems in a realistic fading channel," accepted in IEEE RWW conference, to be published in 2018.

[20] B. Tan, K. Woodbridge and K. Chetty, "A real-time high resolution passive WiFi Doppler-radar and its applications," 2014 International Radar Conference, Lille, 2014, pp. 1-6.

[21] L. G. Barbero and J. S. Thompson, "Rapid prototyping of a fixedthroughput sphere decoder for MIMO systems," 2006 IEEE International Conference on Communications, Istanbul, 2006, pp. 3082-3087.

[22] M. R. Perrett and I. Darwazeh, "Flexible hardware architecture of SEFDM transmitters with real-time non-orthogonal adjustment," 2011 18th International Conference on Telecommunications, Ayia Napa, 2011, pp. 369-374.

[23] R. C. Grammenos, S. Isam and I. Darwazeh, "FPGA design of a truncated SVD based receiver for the detection of SEFDM signals," 2011 IEEE 22nd International Symposium on Personal, Indoor and Mobile Radio Communications, Toronto, ON, 2011, pp. 2085-2090.

[24] P. N. Whatmough, M. R. Perrett, S. Isam and I. Darwazeh, "VLSI architecture for a reconfigurable spectrally efficient FDM baseband transmitter," in IEEE Transactions on Circuits and Systems I: Regular Papers, vol. 59, no. 5, pp. 1107-1118, May 2012

[25] Z. Wu, "Coding and Iterative Detection for Magnetic Recording Channels", Springer US, 2000. 